DOI: $10.24850 /$ j-tyca-2018-05-05

Artículos

\title{
Estimación geoestadística de la distribución espacial de la precipitación media mensual y anual en Nuevo León, México (1930-2014)
}

\author{
Silvio Gustavo Villarreal Macés ${ }^{1}$ \\ Martín Alberto Díaz Viera² \\ ${ }^{1}$ Instituto Nacional de Estadística y Geografía (INEGI), \\ silvio.villarreal@inegi.org.mx \\ ${ }^{2}$ Instituto Mexicano del Petróleo (IMP), mdiazv@imp.mx
}

Autor para correspondencia: Silvio Gustavo Villarreal Macés, silvio.villarreal@inegi.org.mx

\section{Resumen}

Considerando la fuerte variabilidad espacial del fenómeno de la precipitación en el estado de Nuevo León, México, se utilizaron las técnicas geoestadísticas de kriging ordinario y simulaciones estocásticas, con el objetivo de estimar la distribución espacial de la precipitación media anual y para las estaciones de mayor precipitación (primavera y verano). Los datos para el estudio corresponden a 95 estaciones climatológicas, con al menos 25 años de registro en el periodo comprendido de 1930 a 2014, se incluyen datos de estaciones vecinas de la NOAA y estados colindantes a Nuevo León. El uso sistemático de estas técnicas permitió establecer la dependencia espacial de los datos de precipitación, por medio de modelos variográficos isotrópicos y anisotrópicos, los cuales se aplicaron en las estimaciones y simulaciones. Para evaluar la calidad de las estimaciones obtenidas se aplicó la técnica de validación cruzada. Los resultados obtenidos son consistentes con el comportamiento estacional de la precipitación en las regiones climáticas del norte y noreste de México, considerando su variabilidad espacial, y la disposición y calidad de la información de las estaciones climatológicas en el área de estudio.

Palabras clave: geoestadística, estimación espacial, precipitación, kriging, simulaciones, anisotropía, Nuevo León. 
Recibido: 09/06/2017

Aceptado: 03/04/2018

\section{Introducción}

Los campos en los que se requiere conocer la distribución espacial de la precipitación son diversos, entre éstos se encuentran la hidrología, agricultura y climatología; en hidrología, es un insumo importante para el modelado hidrológico, en la predicción de eventos extremos, como crecientes en el flujo superficial de agua e inundaciones, la presencia de lluvia durante el año es muy importante, ya que en cierta medida influye en el sector agrícola (Englehart \& Douglas, 2000), en especial, la agricultura de temporal, ya que es dependiente de la precipitación y por tanto, altamente vulnerable a la variabilidad interanual e interestacional de la misma. Por otro lado, el cambio climático tiene impactos directos sobre el régimen de precipitación, lo cual afecta en la gestión de los recursos hídricos, de ahí la importancia de analizar y estudiar el cambio en el patrón espacial y temporal de la precipitación.

Los datos utilizados para este estudio provienen de la red de estaciones climatológicas de la Comisión Nacional del Agua (CONAGUA), y de la NOAA (The National Oceanic and Atmospheric Administration), para el caso del estado de Nuevo León se encuentran distribuidas irregularmente, y en algunas de estas estaciones, la información disponible es insuficiente para caracterizar la alta variabilidad de la precipitación y su distribución espacial. Por lo tanto, es necesario estimar la precipitación en áreas donde no se dispone de información, utilizando datos de las estaciones vecinas. Varios autores como Goovaerts (1999); Mirás-Avalos, Paz-González, Vidal-Vázquez y SandeFouz (2007); Coulibaly y Becker (2007), han demostrado que los métodos geoestadísticos proporcionan mejores estimaciones de la precipitación, en comparación con otras técnicas.

Los objetivos principales de este estudio son: 
1. Analizar y modelar la variabilidad espacial de la precipitación media mensual y anual.

2. Construir superficies estimadas y de sus errores, utilizando kriging ordinario y simulaciones secuenciales gaussianas para la media anual y los meses de mayor precipitación, agrupados en dos periodos estacionales en Nuevo León, Primavera (febrero $16.8 \mathrm{~mm}$ - mayo 55.6 $\mathrm{mm}$ ) y Verano (junio $64.8 \mathrm{~mm}$ - septiembre $117.7 \mathrm{~mm}$ ).

Evaluar los resultados de las técnicas utilizadas.

\section{Descripción de los datos y área de estudio}

En general, en México, la distribución de precipitación varía en espacio y tiempo; es heterogénea a lo largo del año, geográficamente aumenta en dirección norte-sur, debido a la influencia de la latitud. Del mismo modo, está gobernada en gran medida por la proximidad al Océano Pacífico y Golfo de México (Campos-Aranda, 1998), a la orografía del país y a los rasgos de la circulación atmosférica (García, 2003).

El estado de Nuevo León se localiza en el noreste de México, entre los meridianos $98^{\circ} 26^{\prime}$ y $101^{\circ} 14^{\prime}$ de longitud oeste, y entre los paralelos $23^{\circ} 11^{\prime}$ y $27^{\circ} 49^{\prime}$ de latitud norte (Figura 1 ), cuenta con una superficie de $64000 \mathrm{~km}^{2}$ con clima seco. Son tres las provincias fisiográficas que lo atraviesan de manera parcial, la Sierra Madre Oriental, las grandes llanuras de Norteamérica y la llanura costera del golfo, el rango de altitud oscila entre 70 a 3500 metros. 


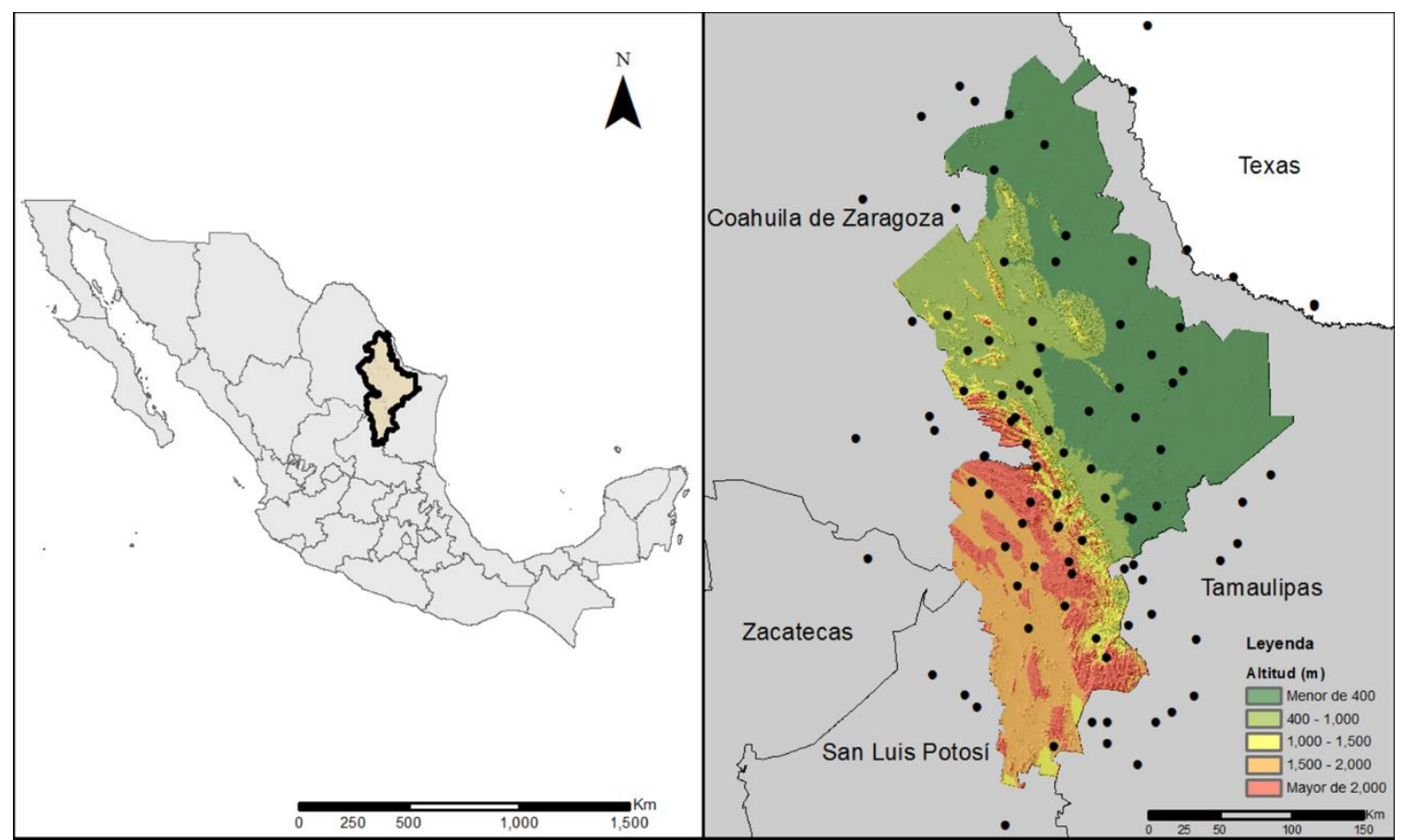

Figura 1. Ubicación del área de estudio y distribución de estaciones climatológicas utilizadas para la estimación de la precipitación en el estado de Nuevo León, México.

Los climas secos y semisecos se distribuyen principalmente en la región nororiental, la cual forma gran parte de la Gran Llanura de Norteamérica; y en la región suroccidental, separada de la primera, por las alturas de la Sierra Madre Oriental. En áreas menores de la región de la sierra, en la zona del centro y sur de la entidad, y en gran parte de la cuenca del Río San Juan se registran los climas semicálidos, templados y semifríos (INEGI, 1986), como se observa en la Figura 2. 


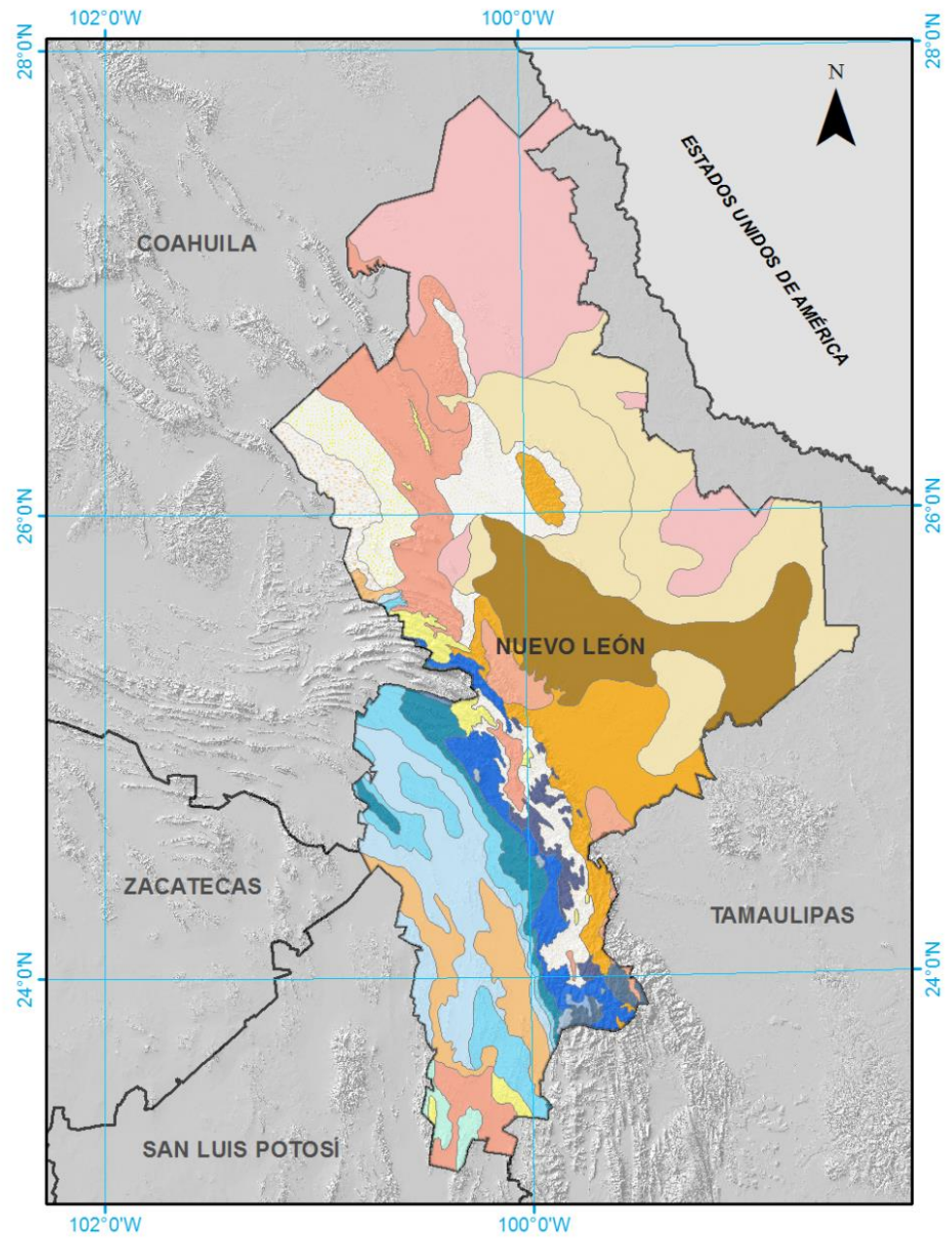

Unidades Climáticas

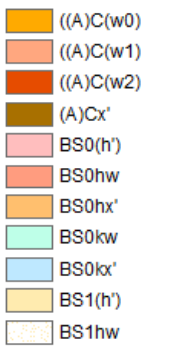

\begin{tabular}{|c|c|}
\hline Semicálido subhúmedo, con lluvias en verano, de menor hume & BS1kw \\
\hline Semicálido subhúmedo, con lluvias en verano, humedad media & BS1kx' \\
\hline Semicálido subhúmedo, con lluvias en verano, más humedo & BWhw \\
\hline Semicálido subhúmedo, con lluvias escasas todo el año & BWhx' \\
\hline Seco muy cálido y cálido, con lluvias en verano & $C(E)(w 1)$ \\
\hline Seco semicálido, con lluvias en ver ano & $C(E)(w 2)$ \\
\hline Seco semicálido, con lluvias escasas todo el año & $C(E) x^{\prime}$ \\
\hline Seco templado, con lluvias en verano & $C($ wo $)$ \\
\hline Seco templado, con lluvias escasas todo el año & $C(w 1)$ \\
\hline Semiseco muy cálido y cálido & $\mathrm{C}(\mathrm{w} 2)$ \\
\hline Semiseco semicálido, con lluvias en verano & $\mathrm{Cx}$ \\
\hline
\end{tabular}

Semiseco templado, con lluvias en verano Semiseco templado, con lluvias escasas todo el año Muy seco semicálido, con lluvias en verano

Muy seco semicálido, con lluvias escasas todo el año Semifrío subhúmedo, con lluvias en verano, humedad media Semifrío subhúmedo, con lluvias en verano, más húmedo Semifrío subhúmedo, con lluvias escasas todo el año Templado subhúmedo, con lluvias en verano, menor humedad Templado subhúmedo, con lluvias en verano, humedad media Templado subhúmedo, con lluvias en verano, mayor humedad Templado subhúmedo, con lluvias escasas todo el año

Figura 2. Tipos de climas de Nuevo León, México.

La precipitación en el estado está dominada por situaciones estacionales y con una gran variabilidad; por ejemplo el área se ve afectada por fuertes lluvias, causadas principalmente por tormentas tropicales y huracanes, debido a su cercanía al Golfo de México y la influencia de 
frentes fríos; en general, la precipitación es escasa, aunque cuenta con regiones que registran lluvias anuales mayores a $1100 \mathrm{~mm}$, la media anual para Nuevo León en el periodo de estudio es de $541 \mathrm{~mm}$.

El periodo de 1930 a 2014 fue seleccionado para la integración de los datos de precipitación acumulados mensual y anualmente, considerando al menos 25 años de registro, para cada una de las estaciones climatológicas utilizadas; 56 se encuentran ubicadas en Nuevo León, y con la finalidad de contar con una mayor cobertura espacial se incorporaron 11 de Coahuila, 17 de Tamaulipas, 4 de San Luis Potosí, una de Zacatecas y 6 de Texas, en Estados Unidos de América; tomando en cuenta el criterio de mantener una distancia promedio menor de 50 $\mathrm{km}$ al límite del estado de Nuevo León, obteniendo un total de 95 estaciones, a partir de las cuales se derivaron las variables PMA, Primavera y Verano, de acuerdo con la Figura 1.

\section{Metodología}

La aplicación de la metodología geoestadística a un conjunto de datos, involucra tres pasos: análisis exploratorio de datos, análisis estructural, y la estimación y/o simulación. En el análisis exploratorio se aplican procesos de control de calidad y criterios para la integración de las series de datos climáticos, aplicando las recomendaciones publicadas por la WMO (World Meteorological Organization) En los documentos, Calculation of monthly and annual 30-year standard normals (WMO, 1989) y Guide to Climatological Practices (WMO, 2011), se analiza la estadística básica de los datos mensuales y anuales integrados.

El análisis estructural consiste en analizar los datos para conocer su variabilidad espacial, evaluar la presencia de anisotropía y determinar los modelos variográficos para cada variable, para posteriormente proceder a validarlos, mediante la técnica de validación cruzada, la tercera etapa es la estimación y simulación, en la cual se obtienen las superficies interpoladas. En este proyecto, se aplicó además del kriging ordinario, la técnica de simulación secuencial gaussiana, la cual consiste en que un nuevo valor simulado que se obtiene a partir de una función de distribución de probabilidad, utilizando valores muestreados y valores 
previamente simulados en una vecindad dada, aplicando una técnica de kriging (Chilès \& Delfiner, 1999).

\section{Resultados}

\section{Análisis Exploratorio}

El control de calidad aplicado a los datos, consistió en la evaluación de los siguientes puntos:

Consistencia espacial. Se actualizaron las coordenadas de 8 estaciones visitadas: 5 de Coahuila $(5016,5032,5035,5048,5049)$ y 3 de Nuevo León (19012, 19054 y 19069), debido a que presentaban inconsistencia en su ubicación.

Formato. Se depuraron registros repetidos y fechas improbables; por ejemplo, en los registros de la NOAA, invariablemente todos los meses, de todos los años tienen un formato de 31 días.

Completitud. Para la integración de los datos anuales, se seleccionaron las 95 estaciones que cumplieron con un periodo de al menos 25 años de registro, considerando el criterio de excluir los meses con más de 5 registros diarios faltantes o más de 3 registros diarios continuos.

La estadística básica para las variables PMA, Primavera y Verano, se describe en la Tabla 1. Las tres variables de precipitación tienen una distribución asimétrica positiva, por lo que es necesario evaluar si en estos casos se presenta el conocido "efecto proporcional", que es una forma particular de heterocedasticidad (la variabilidad de los datos cambia a lo largo del área de estudio), en particular para distribuciones asimétricas positivas, la varianza local se incrementa conforme se aumenta su media local (Goovaerts, 1997), este efecto proporcional 
puede hacer el variograma experimental no interpretable (Grimes \& Pardo-Igúzquiza, 2010).

Tabla 1. Estadística básica de las variables analizadas. DE (desviación estándar), CV (coeficiente de variación).

\begin{tabular}{|c|c|c|c|c|c|c|c|c|c|c|}
\hline Variable & $\begin{array}{c}\text { Media } \\
(\mathbf{m m})\end{array}$ & $\begin{array}{c}\text { DE } \\
(\mathbf{m m})\end{array}$ & $\begin{array}{c}\mathbf{C V} \\
\mathbf{( m m})\end{array}$ & $\begin{array}{c}\text { Min } \\
\mathbf{( m m})\end{array}$ & $\begin{array}{c}\mathbf{Q 1} \\
\mathbf{( m m})\end{array}$ & $\begin{array}{c}\text { Mediana } \\
\mathbf{( m m})\end{array}$ & $\begin{array}{c}\mathbf{Q 3} \\
(\mathbf{m m})\end{array}$ & $\begin{array}{c}\text { Max } \\
(\mathbf{m m})\end{array}$ & Asimetría & Curtosis \\
\hline PMA & 539.2 & 199.8 & 0.4 & 212.7 & 394.5 & 507.6 & 661.9 & 1,110 & 0.77 & 3.2 \\
\hline log PMA & 6.2 & 0.4 & 0.1 & 5.4 & 6.0 & 6.2 & 6.5 & 7.0 & -0.1 & 2.7 \\
\hline Verano & 308.3 & 126.6 & 0.4 & 114.8 & 222.0 & 285.6 & 367.1 & 700.9 & 0.9 & 3.7 \\
\hline $\begin{array}{c}\text { Iog } \\
\text { Verano }\end{array}$ & 5.7 & 0.4 & 0.1 & 4.7 & 5.4 & 5.7 & 5.9 & 6.6 & -0.05 & 2.6 \\
\hline Primavera & 123.5 & 44.8 & 0.4 & 40.6 & 98.5 & 117.7 & 147.7 & 243.1 & 0.5 & 2.9 \\
\hline
\end{tabular}

Para conocer cómo están relacionados los cambios en la varianza local, con los cambios en la media local, es común utilizar gráficas de dispersión calculadas a partir de ventanas móviles (Goovaerts, 1997). Se calcularon las estadísticas para ventanas móviles con un área de 100 $\mathrm{km}^{2}$, con sobreposición de $25 \mathrm{~km}^{2}$; se seleccionaron 18 , que incluyen al menos 10 datos. La sobreposición de las ventanas implica que algunos datos fueron utilizados en repetidas ocasiones para el cálculo de las medias y varianzas locales, esto no tiene relevancia desde el momento en que sólo se quiere establecer si existe o no el efecto proporcional (Isaaks \& Srivastava, 1989), el resultado obtenido para PMA y Verano muestra que, las varianzas y medias locales tienen baja correlación $\left(\rho_{\text {rank }}=0.31\right)$ por lo que se puede asumir la homocedasticidad, en tanto que Primavera presenta un efecto proporcional, debido a una correlación mayor $\left(\rho_{\text {rank }}=0.62\right)$; en la Figura 3 se muestran las gráficas de dispersión. 


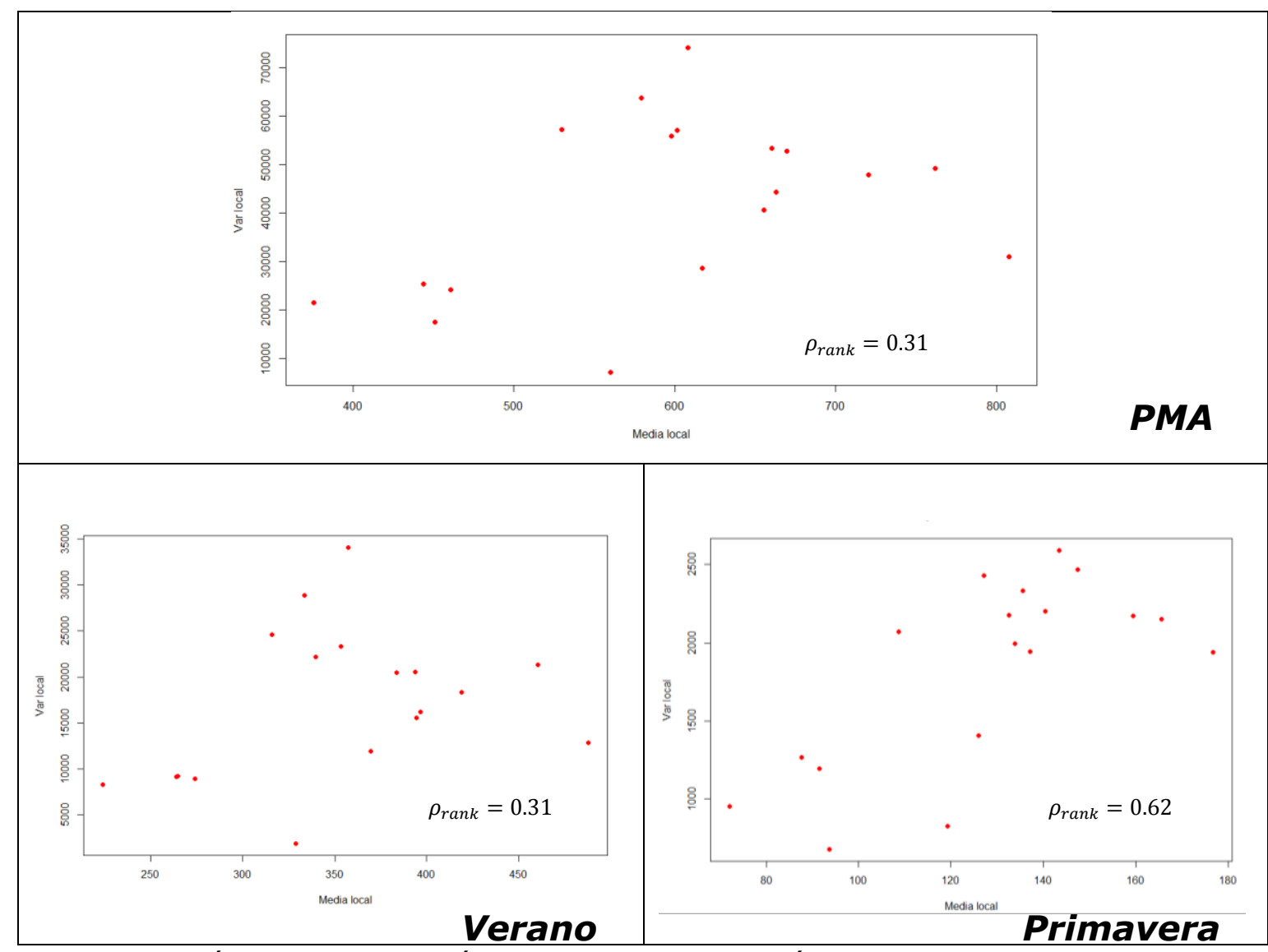

Figura 3. Gráficas de dispersión para 18 ventanas móviles con al menos 10 datos por ventana.

Se sabe que las estaciones climatológicas son ubicadas en lugares donde se cuente con ciertas características para su operación y registro, por lo que existen áreas con mayor concentración (clustering), en particular cuando se combinan el efecto proporcional y el agrupamiento de los datos de la muestra, conducen a serios problemas en la interpretación de los variogramas experimentales, una manera de conocer el agrupamiento y el efecto proporcional es a través de graficar las medias y varianzas locales en función de la distancia (Goovaerts, 1997).

Para el caso de las tres variables se observa un comportamiento que fluctúa alrededor de un valor unitario, concluyendo que las varianzas y las medias no se afectan de manera significativa con la distancia, por lo que no se requiere realizar algún proceso para considerar el efecto de agrupamiento; por ejemplo, usar solo datos regularmente espaciados 
(Goovaerts, 1997). En la gráfica de la Figura 4, sólo se muestran los resultados obtenidos para PMA.

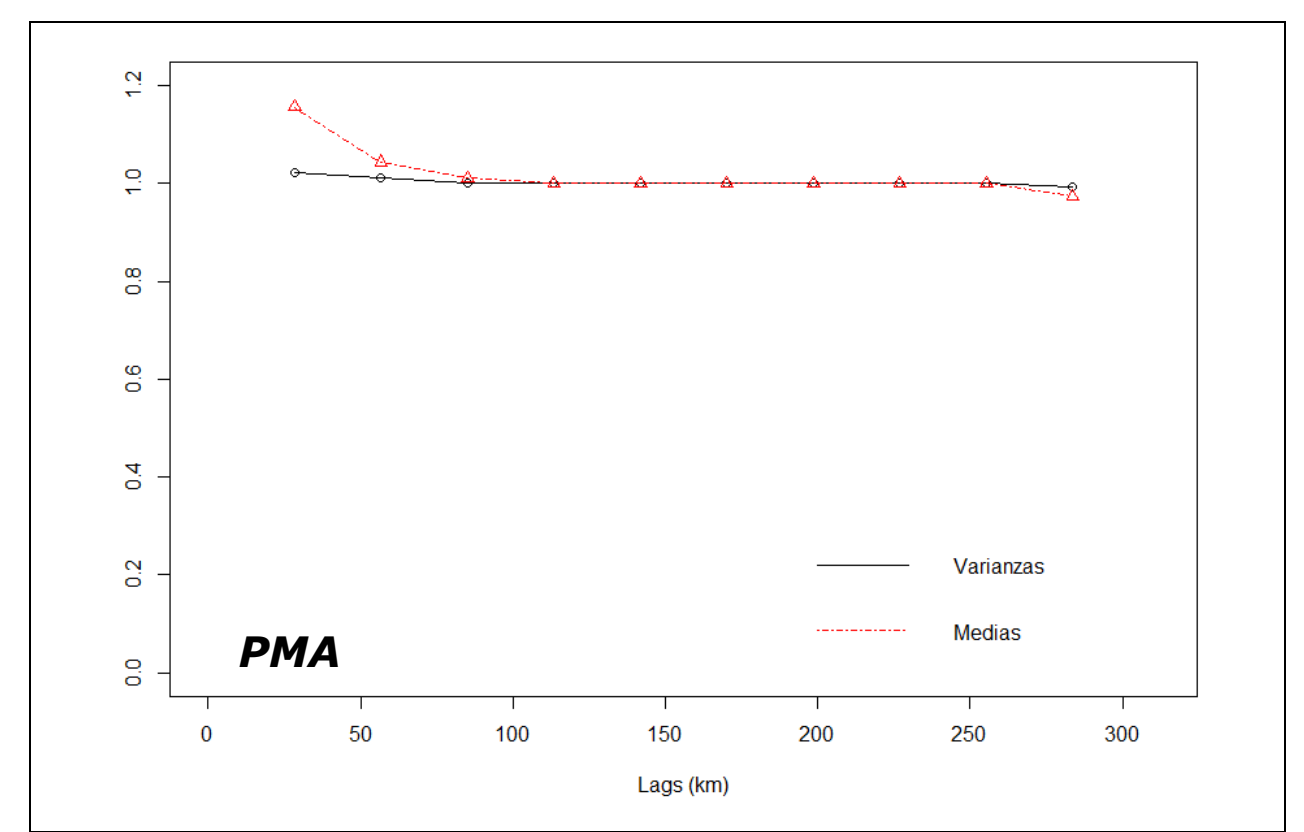

Figura 4. Medias y varianzas como una función de la distancia (lags). Ambos estadísticos son normalizados por sus correspondientes media y varianza global.

\section{Análisis estructural}

Considerando que el grado de asimetría y los datos atípicos afectan de manera directa en el modelado de los variogramas, se aplicó una transformación logarítmica para disminuir el grado de asimetría y analizar el comportamiento de los datos atípicos. La transformación logarítmica utilizada para Primavera no mejoró la distribución, por lo cual no se consideró su aplicación. Las gráficas de la Figura 5, muestran los variogramas experimentales para las variables con asimetría moderada ( $P M A$ y Verano), se incluyó su transformación logarítmica en la misma gráfica, después de reescalar la varianza. Los variogramas logarítmicos parecen menos erráticos, con menor nugget para PMA, por lo tanto, esta variable se modela sin transformación, mientras que Verano sí justifica la transformación, debido a la notable diferencia entre los variogramas. 


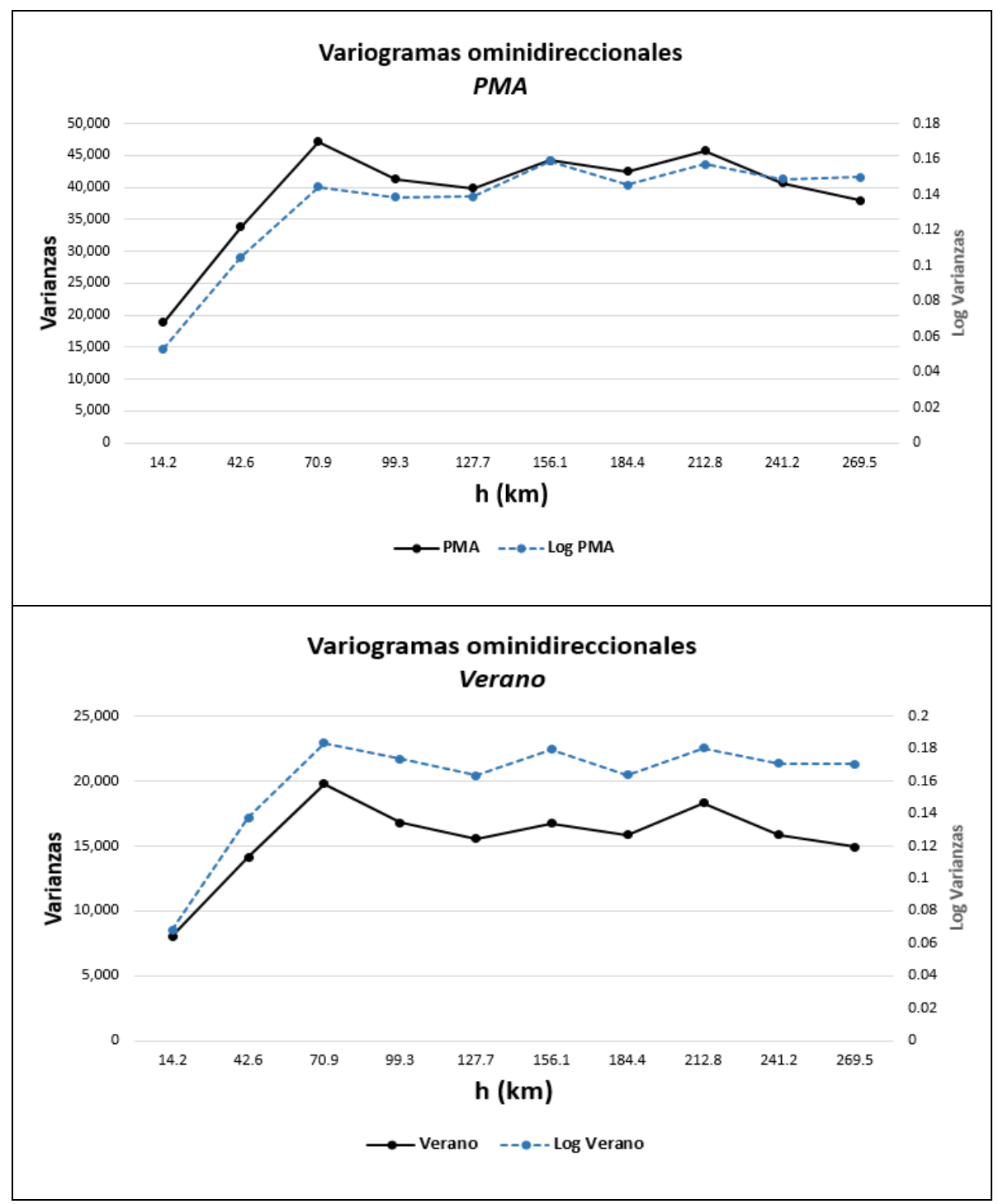

Figura 5. Variogramas experimentales omnidireccionales para PMA y Verano, el variograma de los logaritmos es reescalado a la varianza de los datos originales.

El análisis de anisotropía se realizó inspeccionando los variogramas experimentales en diferentes direcciones: $0^{\circ}(\mathrm{N}-\mathrm{S}), 45^{\circ}(\mathrm{NE}-\mathrm{SO}), 9^{\circ}(\mathrm{E}-$ O) y $135^{\circ}(\mathrm{NO}-\mathrm{SE})$ con una tolerancia angular de \pm 22.5 (Wackernagel, 2003). Como en el área de estudio se encuentra ubicada una porción de la Sierra Madre Oriental, la cual influye en los patrones de la precipitación, se construyeron variogramas en la dirección NO $20^{\circ}$, rumbo asociado a la estructura regional de la sierra citada, así como la dirección perpendicular a ésta $\left(\mathrm{NE} 70^{\circ}\right)$, y adicionalmente se construyó 
el variograma omnidireccional (dirección $0^{\circ}$, con una tolerancia angular de $90^{\circ}$ ), con la ayuda del software gstat (Pebesma \& Wesseling, 1998) y el RGEOESTAD (Díaz, Hernández, \& Méndez, 2012).

De acuerdo con las gráficas de la Figura 6, a partir de los puntos de inflexión de los variogramas experimentales, se aprecian dos escalas de variación espacial en la dirección $\mathrm{NE} 70^{\circ}$ (perpendicular a la estructura de la Sierra Madre Oriental): una con un rango de $75 \mathrm{~km}$ y otra de 125 $\mathrm{km}$ aproximadamente, para una distancia mayor a $125 \mathrm{~km}$, la estructura de los variogramas se vuelven erráticas en esa dirección, debido a la disminución del número de pares que contribuyen a los valores de las varianzas, para el caso de la dirección NE $20^{\circ}$, los variogramas muestran una mayor continuidad espacial (menor varianza) y siguen un patrón similar al del variograma omnidireccional. 


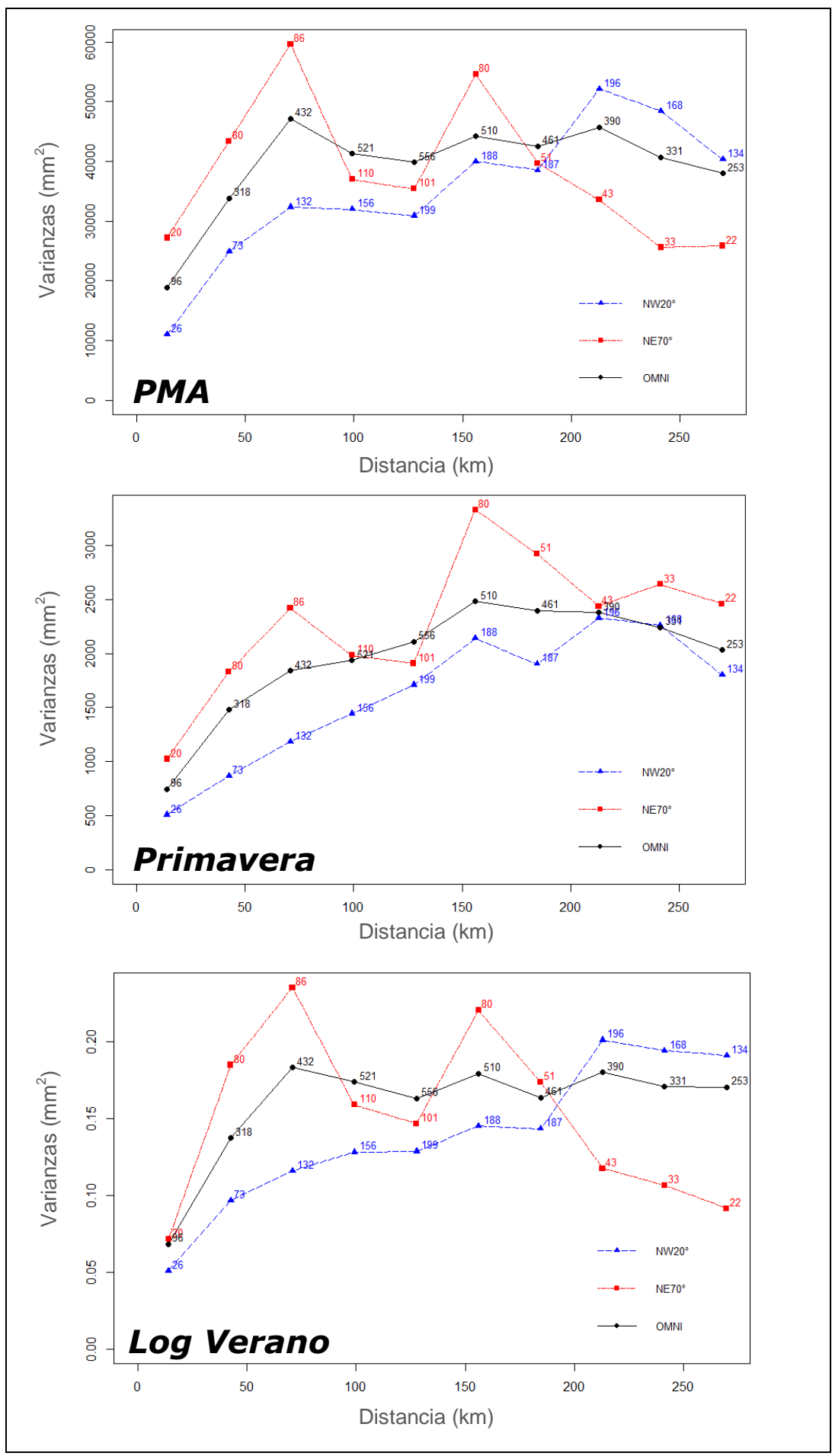

Figura 6. Variogramas $P M A$, Primavera y Log Verano para las direcciones $N E 70^{\circ}, \mathrm{NO}$ $20^{\circ}$ con tolerancia angular de 22.5 líneas discontinuas, el variograma omnidireccional $\left(0^{\circ}\right)$ con tolerancia angular de $90^{\circ}$ en línea continua. Las etiquetas numéricas detallan el número de pares. 
Otra manera de evaluar la anisotropía es a través de la elaboración de lo que se conoce como mapa variográfico (Isaaks \& Srivastava, 1989), en el cual se grafican los valores del variograma experimental y el centro del mapa corresponde al origen del variograma, en la Figura 7 se observa una dirección preferencial hacia el NO-SE para Log Verano y $P M A$, aproximadamente alineada a la que presenta la Sierra Madre Oriental; para el caso de Primavera se aprecia en menor medida esta dirección preferencial.

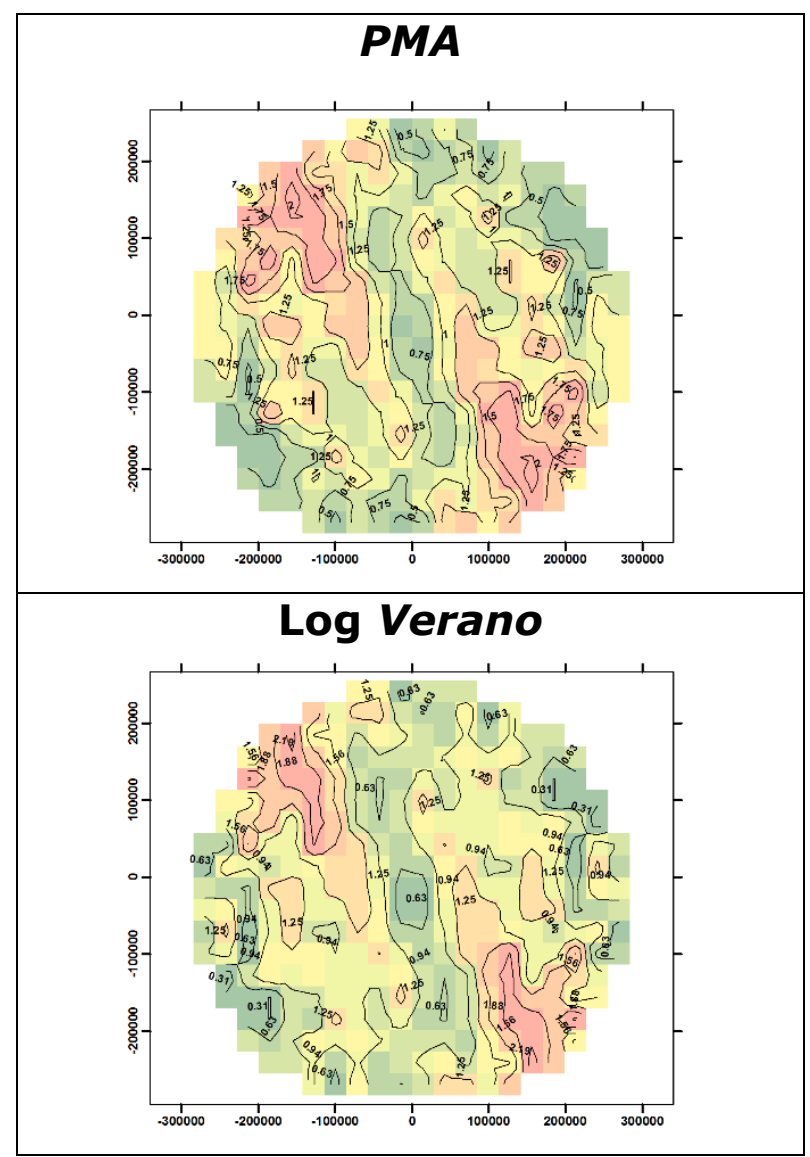




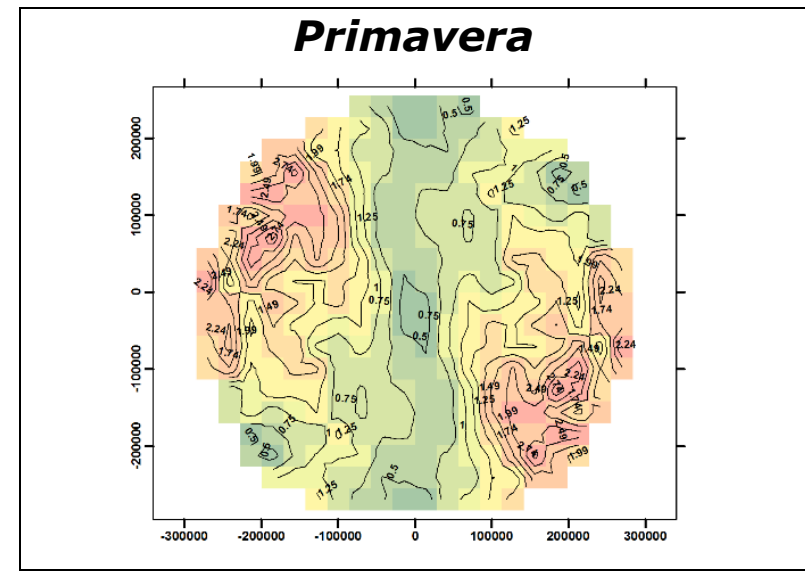

Figura 7. Mapas variográficos, PMA, Log Verano y Primavera, valores estandarizados por la varianza de la muestra.

Tomando en cuenta los resultados arrojados por el mapa variográfico, se utilizaron los modelos anisotrópicos para las variables PMA y log Verano.

Una vez definidas las direcciones de los variogramas se ajustaron modelos teóricos esféricos, con la técnica de mínimos cuadrados, con el apoyo del software RGEOESTAD (Díaz et al., 2012) y gstat (Pebesma \& Wesseling, 1998), se obtuvieron los parámetros variográficos: nugget, sill y rango; en la Tabla 2 se muestran los datos obtenidos para las tres variables analizadas, y la Figura 8 ilustra los modelos esféricos ajustados, incluyendo el omnidireccional. Los modelos ajustados para la dirección NE $70^{\circ}$ no se muestran en este documento.

Tabla 2. Parámetros variográficos NO $20^{\circ}$, NE $70^{\circ}$ y omnidireccional, modelados para las variables Primavera, PMA y Verano logarítmico.

\begin{tabular}{|c|c|c|c|c|c|c|c|c|c|}
\hline Dirección & \multicolumn{3}{|c|}{ NO 20 } & \multicolumn{3}{c|}{ NE 70 } & \multicolumn{3}{c|}{ Omnidireccional } \\
\hline Variable & PMA & Primavera & $\begin{array}{c}\text { Log } \\
\text { Verano }\end{array}$ & PMA & Primavera & $\begin{array}{c}\text { Log } \\
\text { Verano }\end{array}$ & PMA & Primavera & $\begin{array}{c}\text { Log } \\
\text { Verano }\end{array}$ \\
\hline $\begin{array}{c}\text { Nugget } \\
\text { (mm }^{2} \text { ) }\end{array}$ & 6500 & 310 & 0.04 & 16860 & 537 & 0 & 9570 & 398 & 0.03 \\
\hline $\begin{array}{c}\text { Sill } \\
\text { (mm }^{2} \text { ) }\end{array}$ & 41118 & 2140 & 0.18 & 47360 & 2311 & 0.17 & 33850 & 1752 & 0.15 \\
\hline $\begin{array}{c}\text { Rango } \\
(\mathbf{k m})\end{array}$ & 150 & 215 & 230 & 62 & 90 & 44 & 77.2 & 103.6 & 75.7 \\
\hline $\begin{array}{c}\text { Nugget/ } \\
\text { Sill }\end{array}$ & 0.16 & 0.14 & 0.22 & 0.36 & 0.23 & 0 & 0.28 & 0.23 & 0.2 \\
\hline
\end{tabular}


Ciencias $₫$ Agua

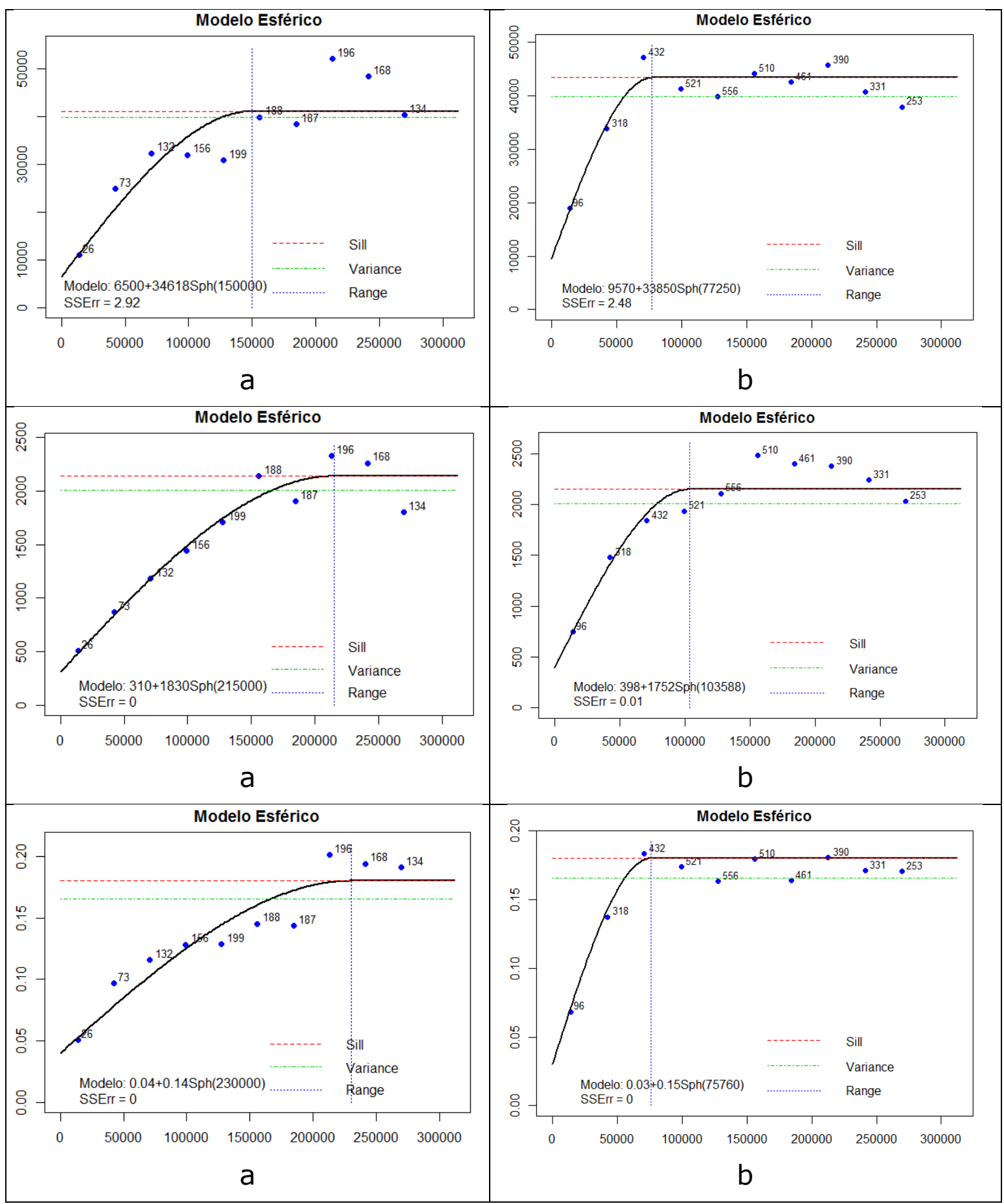

Figura 8. Variogramas direccionales ajustados para PMA, Primavera y Log Verano. a) Dirección NO $20^{\circ}$; b) Omnidireccional. 
Todos los variogramas para la dirección NO $20^{\circ}$, exhiben un comportamiento lineal en el origen, así como pequeños efectos nugget, cuya proporción con respecto al sill se encuentra en el intervalo del $16 \%$ al $23 \%$, y representan una discontinuidad en el origen que es atribuible a errores de medición y a la variación a distancias inferiores del intervalo de muestreo $(28.4 \mathrm{~km})$.

Los variogramas en la dirección NE $70^{\circ}$ se modelaron para obtener el factor de anisotropía $\lambda$, definido como la razón del rango menor (NE $70^{\circ}$ ) y el rango mayor (NO $20^{\circ}$ ).

\section{Validación cruzada}

La técnica de validación cruzada consiste en sacar una observación y estimar su valor con el resto de las observaciones, esto se realiza repetidamente hasta concluir con todas las observaciones.

Los errores son la diferencia entre los valores observados y estimados, y se calculan por medio de la siguiente expresión:

$$
\varepsilon_{i}=Z\left(x_{i}\right)-Z_{i}^{*} \quad i=1, \ldots n
$$

Donde $\varepsilon$ son los errores; $Z$ el valor observado y $Z^{*}$ el estimado.

Existen una serie de indicadores que miden la calidad de la estimación de manera global, en este trabajo se consideran los siguientes:

$$
\begin{aligned}
& M E=\frac{1}{n} \sum_{i=1}^{n} \varepsilon_{i} \\
& R M S E=\sqrt{\frac{1}{n} \sum_{i=1}^{n} \varepsilon_{i}^{2}}
\end{aligned}
$$

La Tabla 3 muestra los resultados de la validación.

Tabla 3. Resultados de los errores obtenidos de la validación cruzada. 


\begin{tabular}{|c|c|c|c|}
\hline \multirow{2}{*}{ VMariable } & Modelo & $\begin{array}{c}\text { ME } \\
\mathbf{( m m )}\end{array}$ & $\begin{array}{c}\text { RMSE } \\
\mathbf{( m m )}\end{array}$ \\
\hline \multirow{2}{*}{ Verano } & anisotrópico & -1.63 & 100.06 \\
\cline { 2 - 4 } & isotrópico & -0.71 & 106.39 \\
\cline { 2 - 4 } & anisotrópico & 6.48 & 71.52 \\
\hline \multirow{2}{*}{ Primavera } & isotrópico & 6.31 & 69.97 \\
\cline { 2 - 4 } & anisotrópico & -0.34 & 22.27 \\
\cline { 2 - 4 } & isotrópico & -0.04 & 23.28 \\
\hline
\end{tabular}

De acuerdo con el RMSE obtenido, el modelo anisotrópico mejoró la estimación de PMA y Primavera, para Verano resultó mejor la estimación del modelo isotrópico, de acuerdo con los resultados obtenidos en la validación cruzada.

\section{Métodos de estimación y simulación}

Los mapas de precipitación estimada y error de la estimación mostrados en la Figura 9, Figura 10 y Figura 11 se realizaron con el software ArcMap, utilizando la extensión geostatistical analyst (ESRI, 2016), utilizando los modelos variográficos ajustados, aplicando las técnicas de kriging ordinario y simulaciones secuenciales gaussianas, estas técnicas son discutidas y analizadas ampliamente en los trabajos de algunos autores como Chilès y Delfiner (1999), Goovaerts (1997), Wackernagel (2003), e Isaaks y Srivastava (1989). 


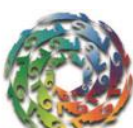

Ciencias
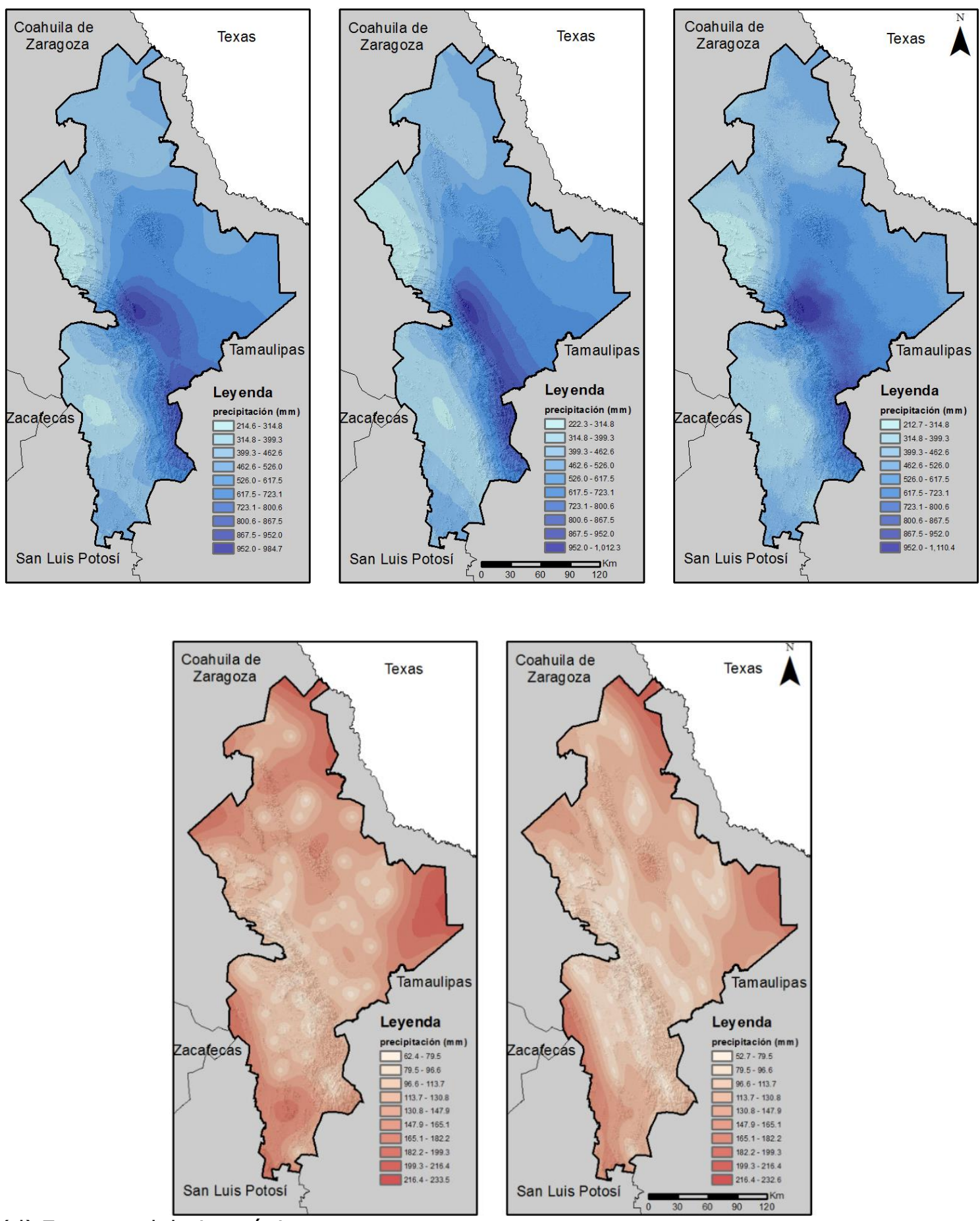

(d) Error modelo isotrópico (e) Error modelo anisotrópico

Figura 9. Mapa de Nuevo León de las estimaciones, simulación y error de la estimación para PMA, en el periodo 1930-2014. 


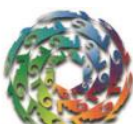

Ciencias ฐ̊Agua

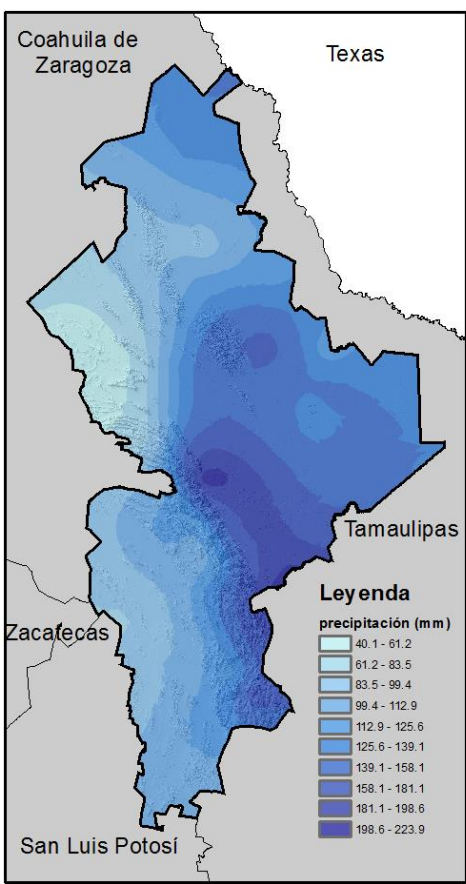

(a) modelo isotrópico

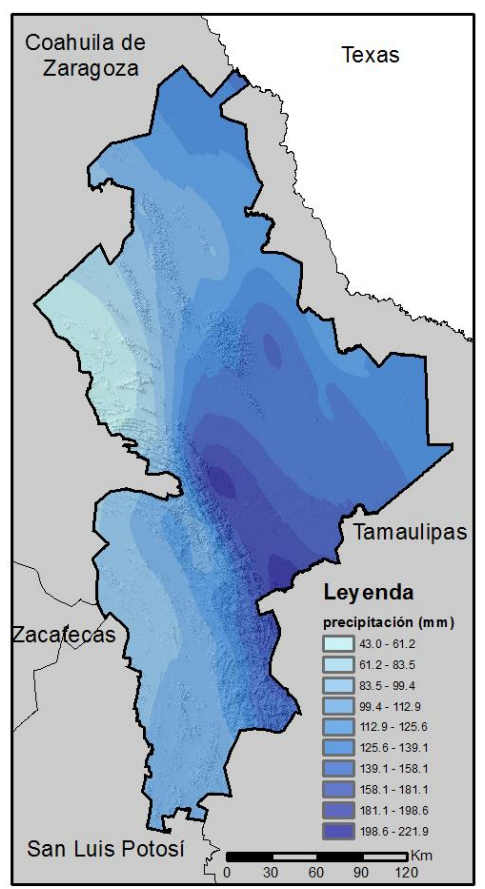

(b) modelo anisotrópico

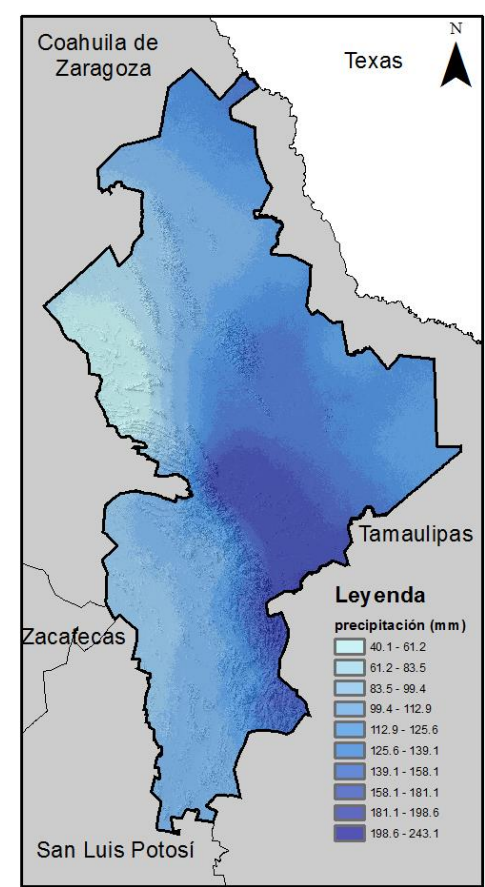

(c) simulación
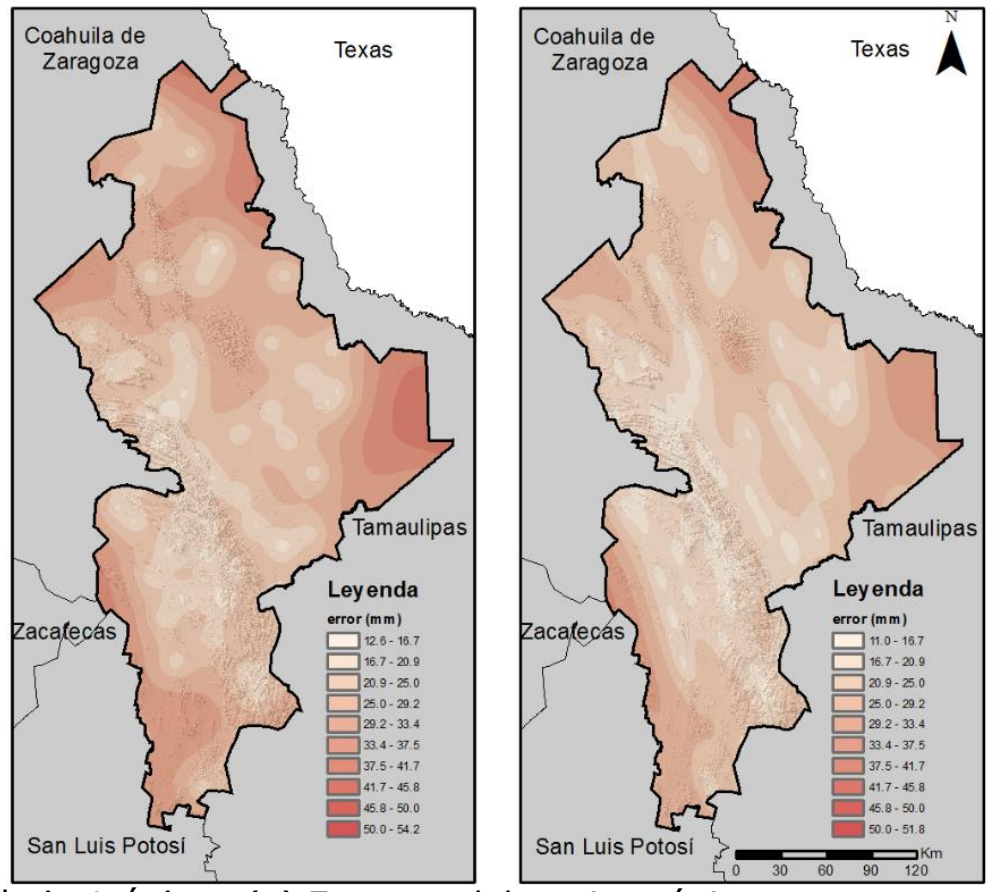

(d) Error modelo isotrópico (e) Error modelo anisotrópico

Figura 10. Mapa de las estimaciones de Nuevo León, simulación y error de la estimación para Primavera, en el periodo 1930-2014. 


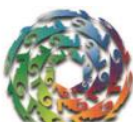

Ciencias

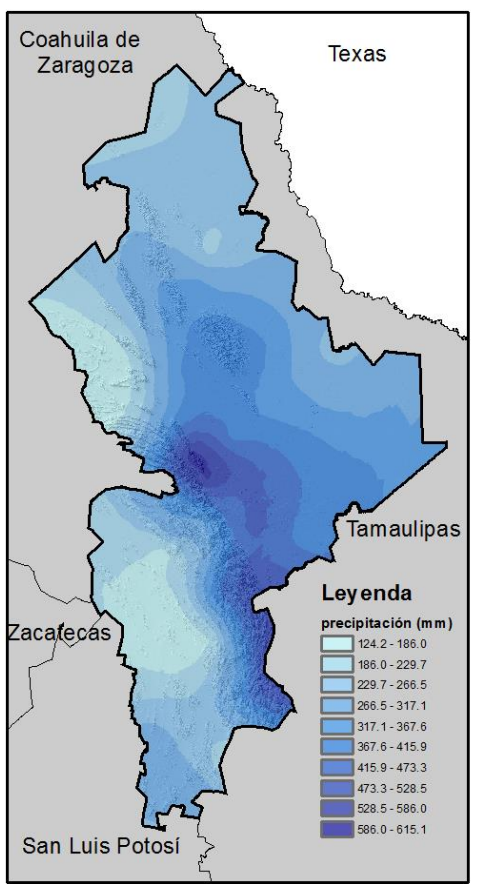

(a) Modelo isotrópico

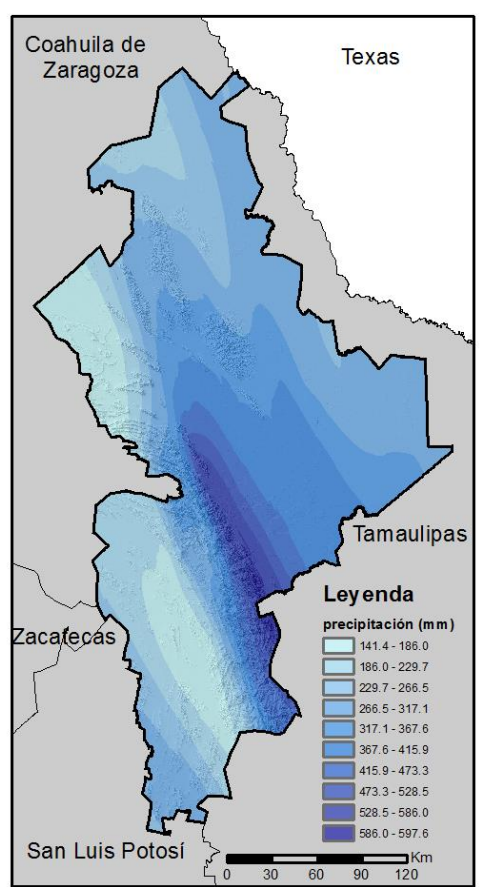

(b) Modelo anisotrópico

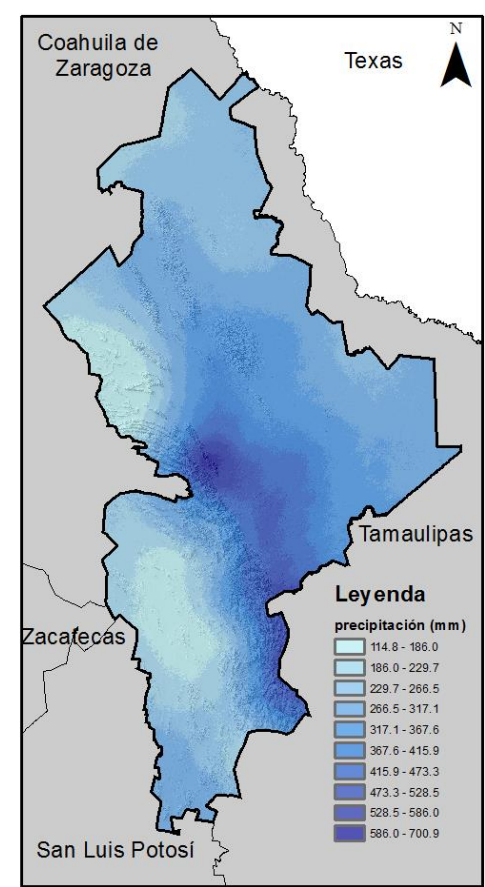

(c) Simulación
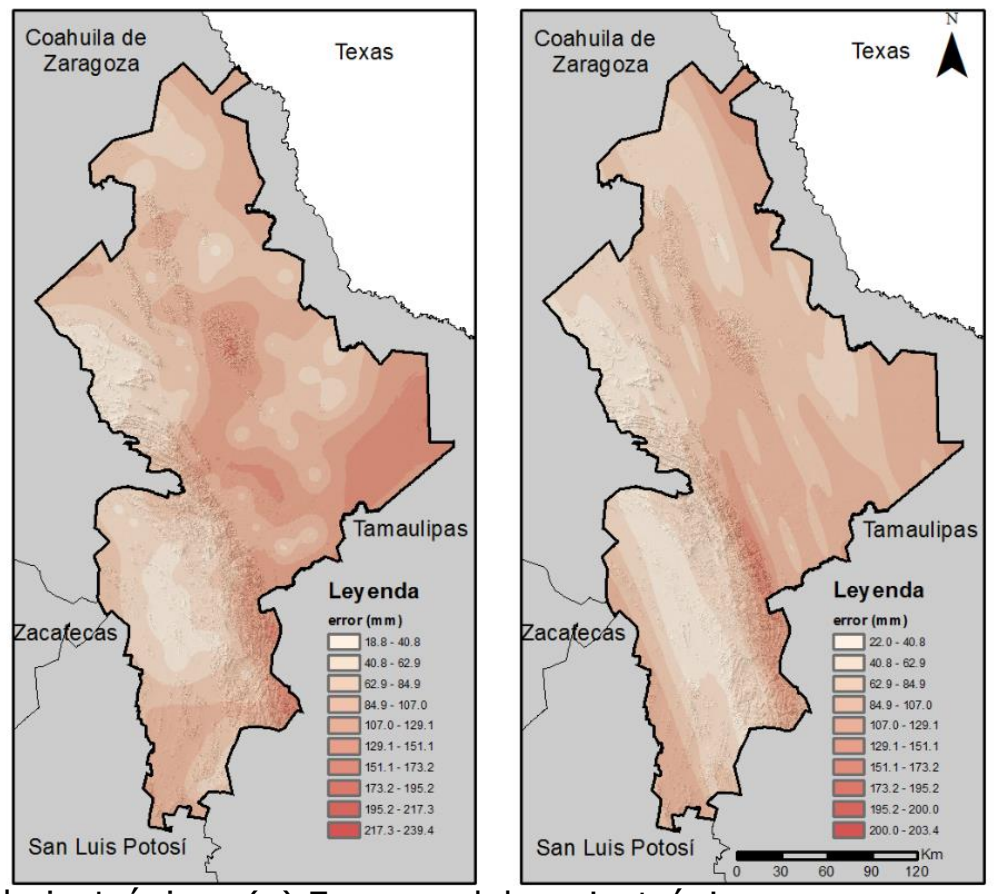

(d) Error modelo isotrópico (e) Error modelo anisotrópico

Figura 11. Mapa de las estimaciones de Nuevo León, simulación y error de la estimación para Verano, en el periodo 1930-2014. 
Para la aplicación de la técnica de simulación secuencial gaussiana, se utilizaron los modelos variográficos isotrópicos, aplicando a los datos una transformación de anamorfosis. Se utilizó el programa de código abierto gstat (Pebesma \& Wesseling, 1998), para realizar 100 simulaciones para cada una de las variables y con el promedio de estas simulaciones se generaron los mapas, utilizando la extensión geostatistical analyst de ArcMap (ESRI, 2016).

En el caso de PMA, el modelo anisotrópico, reprodujo de mejor manera la distribución espacial de los datos de precipitación, enfatizándose la dirección preferencial a la Sierra Madre Oriental, en la parte norte del estado se tienen patrones muy similares entre los tres modelos, y como es de esperarse, para el caso de las simulaciones, produjo fielmente los valores extremos de los datos.

\section{Conclusiones}

El uso de técnicas geoestadísticas, permitió establecer modelos variográficos isotrópicos y anisotrópicos de dependencia espacial para los datos de precipitación, los cuales fueron fundamentales para la aplicación del kriging ordinario y de simulaciones secuenciales gaussianas.

Las simulaciones secuenciales gaussianas definieron, de manera más detallada, los patrones de precipitación estacional para el estado, debido a eventos ciclónicos provenientes del Mar Caribe y Golfo de México, con una dirección dominante NO-SE y con valores superiores a los $40 \mathrm{~mm}$, estos valores contrastan con los resultados de precipitación media mensual, interpolados con técnicas de regresión para la región hidrológica 24 Río Bravo-Conchos, la cual abarca una porción del estado de Nuevo León (Núñez-López et al., 2013), encontrando valores altos para los meses lluviosos, predominantemente en las partes altas de la Sierra Madre Oriental; sin embargo, no se logra identificar con claridad los valores altos de precipitación en las proximidades del área metropolitana de Monterrey, pertenecientes a la porción territorial de la provincia fisiográfica Llanura Costera Golfo Norte, tal vez debido a la cantidad y/o distribución de las estaciones climatológicas utilizadas. 
Por otro lado, con relación a los valores máximos de precipitación media anual obtenidos destaca la región citrícola comprendida por los municipios de Allende, Montemorelos, Hualahuises, General Terán y Linares, esta porción territorial corresponde a la provincia fisiográfica Llanura Costera Golfo Norte, además del Municipio de Santiago y perteneciente a la provincia de la Sierra Madre Oriental, cuyos valores oscilan entre 800 y $1110 \mathrm{~mm}$, acordes con el rango de 800 a 1200 $\mathrm{mm}$, reportado en Vidal (2005).

Considerando los valores obtenidos de RMSE, en la validación cruzada, el uso de los modelos anisotrópicos mejoraron las estimaciones para PMA y Primavera, mientras que para Verano, el modelo isotrópico resultó mejor, en todos los casos es poco significativa la mejoría, por lo que se consideró usar el modelo isotrópico para las simulaciones.

A pesar de que el tiempo de procesamiento es mayor en las simulaciones secuenciales gaussianas se recomienda su uso, debido a que se definieron mejor los patrones de precipitación, que con la utilización de kriging ordinario.

La incorporación de estaciones climatológicas de estados colindantes, incluyendo las de la NOAA en Texas, contribuyeron a una mayor y mejor cobertura espacial, lo que permitió contar con información suficiente para realizar las estimaciones en todo el territorio de Nuevo León.

\section{Agradecimientos}

Al Instituto Nacional de Estadística y Geografía (INEGI), por el apoyo para la realización del presente trabajo.

A Juan Antonio, Diana y Carlos por su valioso apoyo.

\section{Referencias}

Campos-Aranda, D. F. (1998). Procesos del Ciclo Hidrológico. San Luis Potosí, México: Universidad Autónoma de San Luis Potosí.

Chilès, J. P., \& Delfiner, P. (1999). Geostatistics: Modeling Spatial Uncertainty ( ${ }^{\text {nd }}$ ed. $)$. New York, USA: John Wiley \& Sons Inc. 
Coulibaly, M., \& Becker, S. (2007). Spatial interpolation of annual precipitation in South Africa. Comparison and evaluation of methods. Water International, 32(3), 494-502.

Díaz, M., Hernández, V., \& Méndez, J. (2012). RGEOESTAD. México, DF, México: Instituto de Geofísica, Universidad Nacional Autónoma de México. Recuperado http://mmc2.geofisica.unam.mx/gmee/paquetes.html http://mmc2.geofisica.unam.mx/gmee/grupo_md.html

Englehart, P. J., \& Douglas, A. V. (2000). Dissecting the macro-scale variations in Mexican maize yields (1961-1997). Geographical and Environmental Modeling, 4(1), 65-81. DOI: 10.1080/136159300111379

ESRI, Environmental Systems Research Institute. (2016). ArcMapGeostatistical Analyst Extension version 10.5. Inc. Red Lands, California.

García, E. (2003). Distribución de la precipitación en la República Mexicana. Investigaciones Geográficas, 50, 67-76.

Goovaerts, P. (1997). Geostatistics for Natural Resources Evaluation. New York, USA: Oxford University Press.

Goovaerts, P. (1999). Geostatistics in soil science: State-of-the-art and perspectives. Geoderma, 89(1-2), 1-45.

Grimes, D., \& Pardo-Igúzquiza, E. (2010). Geostatistical analysis of rainfall. Geographical Analysis, 42, 136-160.

INEGI, Instituto Nacional de Estadística y Geografía. (1986). Síntesis Geográfica del Estado de Nuevo León. México, DF, México: Secretaría de Programación y Presupuesto.

Isaaks, E. H., \& Srivastava, R. M. (1989). An Introduction to Applied Geostatistics. New York, USA: Oxford University Press.

Mirás-Avalos, J. M., Paz-González, A., Vidal-Vázquez, E., \& Sande-Fouz, P. (2007). Mapping monthly rainfall data in Galicia (NW Spain) using inverse distances and geostatistical methods. Advances in Geosciences, $10,51-57$.

Núñez-López, D., Treviño-Garza, E., Reyes-Gómez, V., Muñoz-Robles, C. Aguirre-Calderón, O., \& Jiménez-Pérez, J. (2013). Interpolación espacial de la precipitación media mensual en la cuenca del río Bravo/Grande. Tecnología y Ciencias del Agua, 4(2), 185-193.

Pebesma, E. J., \& Wesseling, C. G. (1998). Gstat, a Program for Geostatistical Modelling, Prediction and Simulation. Computers \& Geosciences, 24, 17-31. 
Vidal, Z. R. (2005). Las Regiones Climáticas de México. México, DF, México: Instituto de Geografía, Universidad Nacional Autónoma de México.

Wackernagel, H. (2003). Multivariate Geostatistics: An introduction with Applications. Springer-Verlag Berlin Heidelberg New York.

WMO, World Meteorological Organization. (1989). Calculation of Monthly and Annual 30-Year Standard Normal. WDCP-No. 10, WMO-TD/No. 341, Geneva: World Meteorological Organization, 11p.

WMO, World Meteorological Organization. (2011). Guide to Climatological Practices. WMO-No.100, Geneva: World Meteorological Organization, capítulo 3. 